\title{
From RHIC to LHC: A Relativistic Diffusion Approach
}

\author{
Rolf Kuiper and Georg Wolschin \\ Institut für Theoretische Physik der Universität Heidelberg, Philosophenweg 16, D-69120 Heidelberg, Germany
}

Received on 4 October, 2006; revised version received on 6 January, 2007

\begin{abstract}
We investigate stopping and particle production in heavy-ion collisions in a Relativistic Diffusion Model (RDM). Using three sources for particle production, the energy- and centrality dependence of rapidity distributions of net protons, and pseudorapidity distributions of charged hadrons in heavy systems at RHIC energies are precisely reproduced in the analytical model. The transport coefficients are then extrapolated from $\mathrm{Au}+\mathrm{Au}$ at RHIC energies $\left(\sqrt{s_{N N}}=19.6-200 \mathrm{GeV}\right)$ to $\mathrm{Pb}+\mathrm{Pb}$ at LHC energies of $\sqrt{s_{N N}}=5.52 \mathrm{TeV}$. Rapidity distributions for net protons, and pseudorapidity spectra for produced charged particles are obtained and discussed in a three-sources model at LHC energies.
\end{abstract}

Keywords: Diffusion model

\section{INTRODUCTION}

Stopping and particle production in relativistic heavy-ion collisions at the highest energies available at RHIC and LHC offer sensitive tools to test the nonequilibrium-statistical properties of these systems. Analytically soluble nonequilibriumstatistical models [1] not only allow to accurately describe a fairly large amount of phenomena, but also to predict results across the gap in center-of-mass energy from the highest RHIC energy in $\mathrm{Au}+\mathrm{Au}$ collisions of $\sqrt{s_{N N}}=200 \mathrm{GeV}[2,3]$ to the LHC energy of $\sqrt{s_{N N}}=5.52 \mathrm{TeV}$ in $\mathrm{Pb}+\mathrm{Pb}$ collisions.

Net-baryon (more precisely, net-proton) distributions have proven to be sensitive indicators for local equilibration, collective expansion, and deconfinement in heavy relativistic systems [4]. This is reconsidered in the analysis of SPS- and RHIC-results within the Relativistic Diffusion Model (RDM) for three sources in Sect. II. The dependence of the transport coefficients on the center-of-mass energy from AGS via SPS to RHIC is then used for predictions of net-baryon rapidity distributions at LHC.

It is of particular interest in relativistic collisions of heavy systems to determine the fraction of produced particles that attains - or comes very close to - local statistical equilibrium. In the three-sources RDM, these are the particles produced in the midrapidity source. Hence $\mathrm{Au}+\mathrm{Au}$ and $\mathrm{Cu}+\mathrm{Cu}$ pseudorapidity distributions of produced particles are analyzed at RHIC energies from $\sqrt{s_{N N}}=19.6-200 \mathrm{GeV}$, and the transport coefficients and numbers of produced particles as functions of the incident energies are determined in Sect. III.

For several reasonably motivated extrapolations of the transport coefficients a range of resulting distribution functions for produced charged hadrons in $\mathrm{Pb}+\mathrm{Pb}$ collisions at LHC energies is discussed. The conclusions are drawn in Sect. IV.

\section{NET-BARYON RAPIDITY SPECTRA}

In the Relativistic Diffusion Model, the net-baryon rapidity distribution at RHIC energies emerges from a superposition of the beam-like nonequilibrium components that are broadened in rapidity space through diffusion due to soft (hadronic, low $\left.p_{\perp}\right)$ collisions and particle creations, and a near-equilibrium (thermal) component at midrapidity that arises - among other processes - from hard (partonic, high $p_{\perp}$ ) processes, and may indicate local quark-gluon plasma (QGP) formation.

The time evolution of the distribution functions is governed by a Fokker-Planck equation (FPE) in rapidity space [1, 4-7]

$$
\frac{\partial}{\partial t}[R(y, t)]^{\mu}=-\frac{\partial}{\partial y}\left[J(y)[R(y, t)]^{\mu}\right]+D_{y} \frac{\partial^{2}}{\partial y^{2}}[R(y, t)]^{\nu}
$$

with the rapidity $y=0.5 \cdot \ln ((E+p) /(E-p))$. The rapidity diffusion coefficient $D_{y}$ that contains the microscopic physics accounts for the broadening of the rapidity distributions due to interactions and particle creations, and it is related to the drift term $J(y)$ by means of a dissipation-fluctuation theorem (Einstein relation) which is used to actually calculate $D_{y}$ in the weak-coupling limit [1]. The drift $J(y)$ determines the shift of the mean rapidities towards the central value, and linear and nonlinear forms have been discussed.

Here we use $\mu=1$ (due to norm conservation) and $q=$ $2-v=1$ corresponding to the standard FPE, and a linear drift function

$$
J(y)=\left(y_{e q}-y\right) / \tau_{y}
$$

with the rapidity relaxation time $\tau_{y}$, and the equilibrium value $y_{e q}$ of the rapidity $[1,8]$. This is the so-called UhlenbeckOrnstein process, applied to the relativistic invariant rapidity for the three components $R_{k}(y, t) \quad(k=1,2,3)$ of the distribution function $[1,4,7]$.

Since the equation is linear, a superposition of the distribution functions [1, 4] emerging from $R_{1,2}(y, t=0)=\delta\left(y \mp y_{b}\right)$ and $R_{3}(y, t=0)=\delta\left(y-y_{e q}\right)$ with mean values and variances obtained analytically from the moments equations yields the exact solution.

With an effective diffusion coefficient $D_{y}^{e f f}$ that includes not only the random behaviour of the particles, but also the effect of expansion, the available net-proton data for central $\mathrm{Au}+\mathrm{Au}$ collisions at AGS, and $\mathrm{Pb}+\mathrm{Pb}$ at SPS [9] can be reproduced precisely with only two sources in the RDM. The corresponding rapidity width coefficient is

$$
\Gamma_{y}^{e f f}=\left[8 \cdot \ln (2) \cdot D_{y}^{e f f} \cdot \tau_{y}\right]^{1 / 2} .
$$




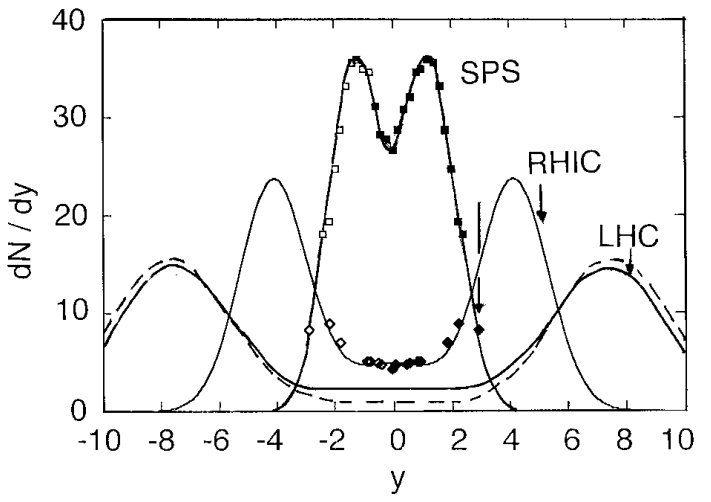

FIG. 1: Net-proton rapidity spectra in the Relativistic Diffusion Model (RDM), solid curves: Transition from the double-humped shape at SPS energies of $\sqrt{s_{N N}}=17.3 \mathrm{GeV}$ to a broad midrapidity valley in the three-sources model at RHIC $(200 \mathrm{GeV})$ and LHC (5.52 TeV). See [11] for details.

Extending the model to RHIC energies of $200 \mathrm{GeV}$, one finds [4] that within the linear approach with $q=1$ and two sources, it is impossible to reproduce the BRAHMS netproton data [2] due to the high midrapidity yield.

It has therefore been proposed in [4] that an expanding midrapidity source emerges. With this conjecture, the RHIC data can be interpreted rather precisely in the linear $q=1$ framework. A fraction of $Z_{e q} \simeq 22$ net protons (55 net baryons) near midrapidity reaches local statistical equilibrium in the longitudinal degrees of freedom, Fig. 2. The variance of the equilibrium distribution $R_{e q}(y)$ at midrapidity is broadened as compared to the Boltzmann result due to collective (multiparticle) effects. This corresponds to a longitudinal expansion (longitudinal flow) velocity of the locally equilibrated subsystem as in hydrodynamical descriptions. Here we obtain the expansion velocity as proposed in [10].

The complete solution of (1) in the $q=1$ case is a linear superposition of nonequilibrium and local equilibrium distributions. The net-baryon rapidity distribution becomes

$$
\frac{d N\left(y, t=\tau_{\text {int }}\right)}{d y}=N_{1} R_{1}\left(y, \tau_{\text {int }}\right)+N_{2} R_{2}\left(y, \tau_{\text {int }}\right)+N_{e q} R_{e q}^{\text {loc }}(y)
$$

with the interaction time $\tau_{\text {int }}$ (total integration time of the differential equation). The number of net baryons (here: net protons) in local equilibrium near midrapidity is $N_{e q}$, and $N_{1}+N_{2}+N_{e q}$ is equal to the total number of net baryons. This yields a good representation of the BRAHMS data [2], as was already emphasized in [10]. Here the integration is stopped at the value of $\tau_{\text {int }} / \tau_{y}$ that yields the best agreement with the data.

Based on the results for net protons from AGS to RHIC energies, the diffusion-model parameters have been extrapolated as functions of the center-of-mass energy to LHC energies of $\sqrt{s_{N N}}=5.52 \mathrm{TeV}$. This allows to obtain a first approximation

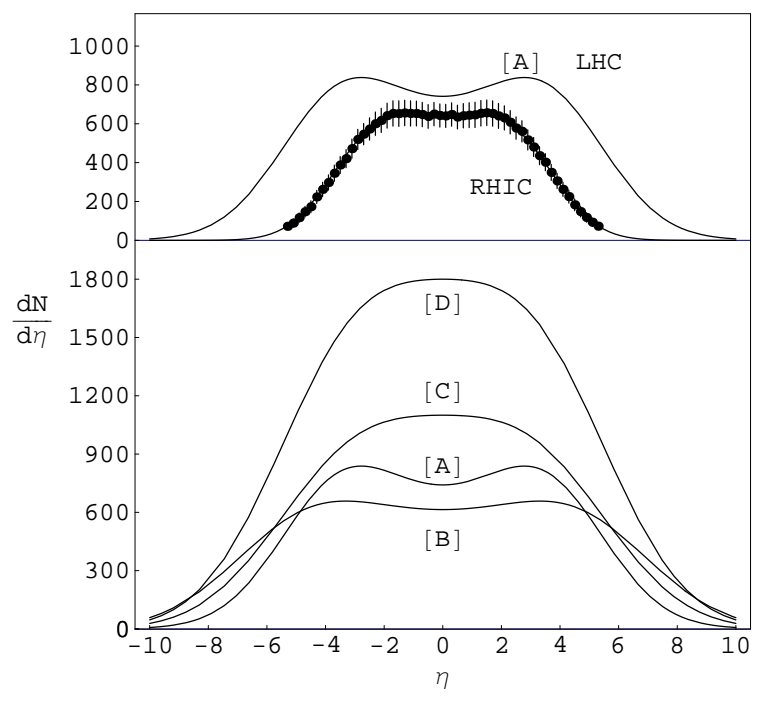

FIG. 2: Produced charged hadrons for central $\mathrm{Au}+\mathrm{Au}$ collisions at RHIC in the RDM compared with $200 \mathrm{~A} \mathrm{GeV} \mathrm{PHOBOS} \mathrm{data} \mathrm{[3],}$ and diffusion-model extrapolation to $\mathrm{Pb}+\mathrm{Pb}$ at LHC energies. See [11] for curves $[\mathrm{A}]$ to $[\mathrm{D}]$ at $\mathrm{LHC}$ energies.

of the net-proton distribution at LHC energies as shown in Fig. 1 together with the SPS- and RHIC-results. Here, the solid curve is for a midrapidity source with a particle content of $14 \%$ as at the highest RHIC energy, whereas the dashed curve corresponds to a particle content of only $7 \%$.

Due to the small value of $\tau_{\text {int }} / \tau_{y}=0.14$ that is obtained from the extrapolation [10], the distribution functions at LHC energies extend well beyond the initial beam rapidity shown by an arrow. Hence, this result will have to be corrected at large rapidities since it is likely to violate kinematic constraints.

\section{PRODUCED CHARGED HADRONS IN THE RDM}

The Relativistic Diffusion Model [1] in its linear form with explicit treatment of the collective expansion [4], or in its nonlinear version with implicit consideration of the collective effects, is also suitable for the description and prediction of rapidity distributions of produced charged hadrons.

If particle identification is not available, one has to convert the results to pseudorapidity space, $\eta=-\ln [\tan (\theta / 2)]$ with the scattering angle $\theta$. The conversion from $y-$ to $\eta-$ space of the rapidity density is performed through the Jacobian

$$
J\left(\eta,\langle m\rangle /\left\langle p_{T}\right\rangle\right)=\cosh (\eta) \cdot\left[1+\left(\langle m\rangle /\left\langle p_{T}\right\rangle\right)^{2}+\sinh ^{2}(\eta)\right]^{-1 / 2} .
$$

The average mass $\langle m\rangle$ of produced charged hadrons in the central region is approximated by the pion mass $m_{\pi}$, and a mean transverse momentum $\left\langle p_{T}\right\rangle=0.4 \mathrm{GeV} / \mathrm{c}$ is used.

In the linear two-sources version, the RDM had been applied to pseudorapidity distributions of produced charged hadrons in $\mathrm{Au}+\mathrm{Au}$ collisions at RHIC energies of $130 \mathrm{GeV}$ 
and $200 \mathrm{GeV}$ by Biyajima et al. [7]. However, it soon turned out from the above net-proton results [4], and from general considerations, that an additional midrapidity source is required $[8,12]$.

To allow for an extrapolation of our results for symmetric systems to LHC energies, RDM-calculations for $\mathrm{Cu}+\mathrm{Cu}$ and $\mathrm{Au}+\mathrm{Au}$ collisions at RHIC energies from $19.6 \mathrm{GeV}$ via 62.4 $\mathrm{GeV}, 130 \mathrm{GeV}$ to $200 \mathrm{GeV}$ are performed as described in [11, 14].

The diffusion-model result at LHC energies with extrapolated transport parameters [11] and 50\% particle content in the central source is shown in Fig. 2, curve $[A]$. The RHIC result for $200 \mathrm{GeV}$ is also displayed.

Diffusion-model results using the extrapolations of the number of produced particles at midrapidity given by other authors have also been obtained. In particular, curve $[C]$ uses a logarithmic extrapolation of the midrapidity value that yields $\mathrm{dN} / \mathrm{d} \eta \simeq 1100$ [13], whereas the saturation model [13] predicts $\mathrm{dN} / \mathrm{d} \eta \simeq 1800$ at midrapidity, with the resulting diffusion-model distribution $[D]$. The forthcoming LHC data are likely to be in between the cases $[A]$ and $[D][11]$.

\section{CONCLUSION}

Net-proton and charged-hadron distributions in collisions of heavy systems at SPS and RHIC energies have been de- scribed in a Relativistic Diffusion Model (RDM) for multiparticle interactions. Analytical results for the rapidity distribution of net protons in central collisions are found to be in good agreement with the available data. An extrapolation of the rapidity distributions for net protons to LHC energies has been performed. The precise number of particles in the midrapidity source remains uncertain at LHC energies and will have to be determined from experiment.

At RHIC - and most likely at LHC -, a significant fraction of the net protons (about 14 per cent at $200 \mathrm{GeV}$ for $\mathrm{Au}+\mathrm{Au}$ ) reaches local statistical equilibrium in a fast and discontinuous transition which may indicate parton deconfinement.

In the three-sources RDM, pseudorapidity distributions of produced charged hadrons have been calculated [11] . The diffusion-model parameters have been optimized in a $\chi^{2}$ minimization with respect to the available PHOBOS data in $\mathrm{Au}+\mathrm{Au}$ and $\mathrm{Cu}+\mathrm{Cu}$ at RHIC. Excellent results for the energy dependence of the distribution functions have been obtained.

In an extrapolation to LHC energies, the pseudorapidity distribution for produced charged hadrons has been calculated. Here the essential parameters relaxation time, diffusion coefficients or widths of the distribution functions of the three sources, and number of particles in the local equilibrium source will have to be adjusted once the ALICE data for $\mathrm{Pb}+$ $\mathrm{Pb}$ have become available in 2009 .
[1] G. Wolschin, Eur. Phys. J. A 5, 85 (1999); Europhys. Lett. 47, 30 (1999); Phys. Rev. C 69, 024906 (2004).

[2] I.G. Bearden et al., BRAHMS Collaboration, Phys. Rev. Lett. 93, 102301 (2004).

[3] B.B.Back et al., PHOBOS Collaboration, Phys. Rev. Lett. 91, 052303 (2003).

[4] G. Wolschin, Phys. Lett. B 569, 67 (2003).

[5] W.M. Alberico, A. Lavagno, and P. Quarati, Eur. Phys. J. C 12, 499 (2000).

[6] M. Rybczyński, Z. Włodarczyk, and G. Wilk, Nucl. Phys. B (Proc. Suppl.) 122, 325 (2003).

[7] M. Biyajima, M. Ide, T. Mizoguchi, and N. Suzuki, Prog. Theor. Phys. 108, 559 (2002).
[8] G. Wolschin, M. Biyajima, T. Mizoguchi, and N. Suzuki, Phys. Lett. B 633, 38 (2006); Annalen Phys. 15, 369 (2006).

[9] H. Appelshäuser et al., NA49 Collaboration, Phys. Rev. Lett. 82, 2471 (1999).

[10] G. Wolschin, Europhys. Lett. 74, 29 (2006).

[11] R. Kuiper and G. Wolschin, Annalen Phys. 16, 67 (2007).

[12] M. Biyajima, M. Ide, M. Kaneyama, T. Mizoguchi, and N. Suzuki, Prog. Theor. Phys. Suppl. 153, 344 (2004).

[13] N. Armesto, C. Salgado, and U. Wiedemann, Phys. Rev. Lett. 94, 022002 (2005).

[14] R. Kuiper, Diploma thesis, Universität Heidelberg (2006). 